\title{
Effet Stener, un diagnostic échographique simple
}

\section{Stener Lesion: a Simple Ultrasound Diagnosis}

\section{Tourdias}

Reçu le 26 avril 2018; accepté le 29 mai 2018

(C) SFMU et Lavoisier SAS 2018

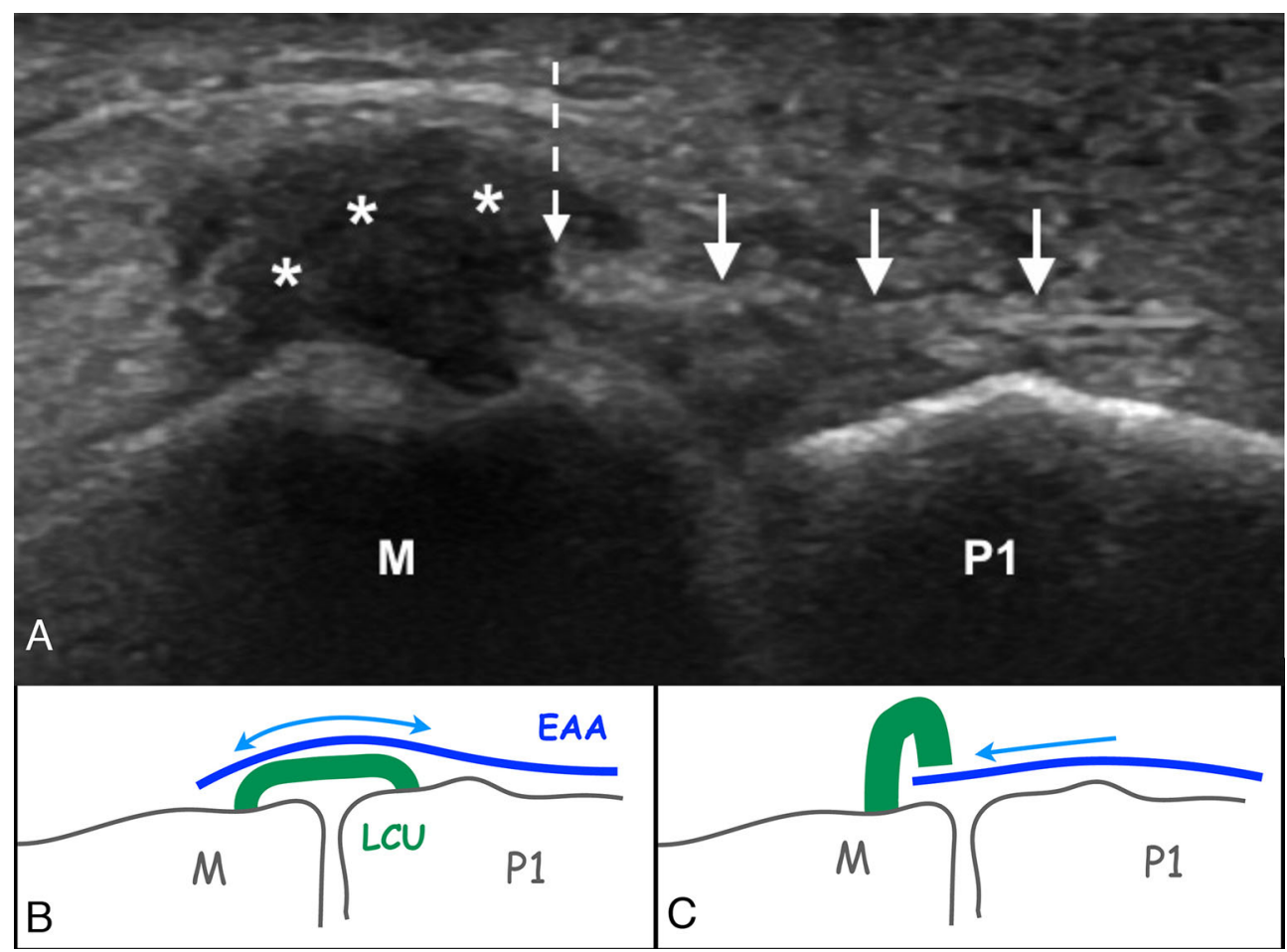

Fig. 1 Aspects échographique et schématique de l'effet Stener à partir de coupes longitudinales du versant ulnaire de la métacarpophalangienne du pouce (MCP). A. Effet Stener échographique typique : avulsion distale du faisceau principal du ligament collatéral ulnaire (LCU) qui apparaît épaissi et rétracté en regard de la tête du métacarpien sous la forme d'un nodule hétérogène globalement hypoéchogène (astérisques) au sein duquel est incarcéré le bord libre proximal (flèche en pointillé) de l'expansion aponévrotique de l'adducteur (EAA) qui ne recouvre plus le ligament (flèches). B. Schéma d'une MCP physiologique : le LCU intact est totalement surmonté de l'expansion aponévrotique de l'adducteur du pouce (EAA) qui glisse librement sur toute sa longueur en échoscopie (double flèche). C. Effet Stener schématisé $\mathrm{M}: 1^{\mathrm{er}}$ métacarpien; $\mathrm{P} 1$ : phalange proximale du $1^{\mathrm{er}}$ rayon

Un patient de 40 ans s'est présenté au deuxième jour d'un traumatisme indirect de l'articulation métacarpophalangienne (MCP) du pouce gauche en hyperextension forcée.

\footnotetext{
D. Tourdias $(\square)$

Consultations posturgences, médecine et traumatologie du sport, centre hospitalier Sud Gironde, rue Paul-Langevin,

F-33210 Langon, France

e-mail : tourdiasdamien@yahoo.fr
}

Il existait une tuméfaction en regard de la MCP avec une douleur exquise à la palpation de sa face ulnaire. Les radiographies standard n'objectivaient aucune fracture. Le testing ligamentaire semblait retrouver une laxité pathologique en valgus forcé. L'examen échographique a permis d'identifier clairement une rupture totale du ligament collatéral ulnaire (LCU) compliquée d'un effet Stener (Fig. 1A) : l'expansion aponévrotique de l'adducteur du pouce (EAA) était interposée entre le LCU et son insertion phalangienne avulsée, 
empêchant ainsi toute cicatrisation spontanée. Il s'agissait donc d'une entorse grave, car potentiellement invalidante, et le patient a ainsi bénéficié le lendemain d'une réinsertion chirurgicale.

L'entorse de la MCP du pouce est fréquente, souvent méconnue et considérée comme un diagnostic difficile [1]. Or, il est indispensable de distinguer les lésions bénignes du LCU (c'est-à-dire sans interposition), dont le traitement est orthopédique, des lésions graves avec effet Stener toujours chirurgicales. L'examen clinique est peu fiable pour faire cette distinction [1]. Seule l'imagerie permet avec certitude de confirmer la présence d'un effet Stener. Si l'IRM peut mettre en évidence cette lésion, l'échographie devrait lui être préférée en première intention, car à la fois plus accessible, plus rapide et moins onéreuse tout en autorisant une étude dynamique. Pour ce faire, il est nécessaire d'utiliser une sonde haute fréquence (12 à $17 \mathrm{MHz}$ ) et que l'opérateur soit formé. Une manœuvre dynamique simple et indolore permet de faciliter le diagnostic échographique d'effet Stener [2]. Elle consiste à imprimer de petits mouvements passifs de flexion-extension à l'interphalangienne du pouce tout en observant en échoscopie les rapports entre le LCU et l'EAA qui normalement doit glisser sur toute la face superficielle du ligament (Fig. 1B). Lors d'une rupture du LCU, ce glissement est toujours possible, sauf en cas d'effet Stener où l'EAA ne recouvre plus le LCU et se retrouve incarcérée au sein du moignon ligamentaire rétracté (Fig. 1A et 1C). Seul un traitement chirurgical, idéalement dans les 10 à 12 jours, permet alors d'obtenir une cicatrisation ligamentaire efficace.

\section{Références}

1. Lark ME, Maroukis BL, Chung KC (2017) The Stener lesion: historical perspective and evolution of diagnostic criteria. Hand 12:283-9

2. Bordet B, Borne J, Fantino O, Pialat JB (2009) Échographie de l'entorse du ligament collatéral ulnaire (LCU) de l'articulation métacarpophalangienne (MCP) du pouce : une nouvelle manœuvre dynamique pour visualiser l'effet Stener. J Radiol 90:217-20 